\title{
Prostatakarzinom: Abirateron verlängert Gesamtüberleben
}

- Heute ist bekannt, dass die Tumorzellen beim metastasierten Prostatakarzinom auch im vermeintlich hormonrefraktären Stadium hormonabhängig bleiben können. Daher hat sich inzwischen die Bezeichnung kastrationsresistentes Prostatakarzinom (CRPC) durchgesetzt. Zu möglichen Mechanismen der Kastrationsresistenz gehören Veränderungen des Androgenrezeptors, die dazu führen, dass dieser besonders sensitiv reagiert oder durch andere Substanzen oder Ligandenunabhängig aktiviert wird. Zudem persistiert die Androgenproduktion trotz konventioneller antihormoneller Therapie, da sie auch in den Nebennieren und den Tumorzellen stattfinden kann. Mit neuen Therapieansätzen werde daher versucht, zum Beispiel jegliche Produktion von Testosteron zu unterbinden, erklärte Prof. Jürgen Gschwend, München, beim diesjährigen DGU-Kongress.
Eine dieser Neuentwicklungen ist Abirateronacetat (Zytiga $\left.{ }^{\circledR}\right)$; sein aktiver Metabolit Abirateron unterdrückt die Androgenproduktion effektiv nicht nur in den Hoden, sondern auch in den Nebennieren und im Tumorgewebe, indem er das Enzym CYP17 selektiv blockiert, das zwei wichtige Schritte in der AndrogenBiosynthese katalysiert. Der erste steroidale, orale Androgen-Biosynthese-Inhibitor ist indiziert in Kombination mit Prednison/Prednisolon beim metastasierten CRPC, das während oder nach einer Docetaxel-haltigen Chemotherapie progredient verläuft. Die Zulassung basiert auf einer Phase-III-Studie, in der 1.195 Patienten mit mCRPC nach Versagen einer Docetaxel-haltigen Chemotherapie Abirateronacetat oder Placebo - jeweils mit Prednison/Prednisolon - erhalten haben [de Bono JS et al. 2011]. Abirateronacetat verlängerte versus Placebo das Gesamtüberleben signifikant (median 15,8 vs. 11,2 Monate, $\mathrm{p}<$ 0,0001) [9]. Bei den sekundären Endpunkten schnitt Abirateronacetat ebenfalls signifikant besser ab. Auch war der Anteil der Patienten mit reduziertem Tumorschmerz größer (44\% vs. $27 \%, p=0,002$ ) und die Dauer bis zum Auftreten von skelettalen Ereignissen länger ( 301 vs. 150 Tage, $p=0,0006$ ). Resultierend aus dem Wirkmechanismus traten unter Abirateronacetat mit Prednison/Prednisolon häufiger Mineralkortikoid-bedingte unerwünschte Ereignisse auf (Flüssigkeitsretention, Hypertonie, Hypokaliämie). Diese seien jedoch in der Regel gut handhabbar, so Prof. Kurt Miller, Berlin. Die Häufigkeit der meisten unerwünschten Ereignisse lag auf Niveau der Kontrollgruppe .

Nach Informationen von

Janssen-Cilag, Neuss

\section{Überaktive Blase: Anticholinerge Therapie beeinflusst nicht die Kognition}

— Die Behandlung der überaktiven Blase (OAB) mit Antimuskarinika als Therapie der ersten Wahl ist bei alten Menschen problematisch, weil die Substanzen aufgrund ihrer Lipophilie gut liquorgängig sind und kognitive Störungen bis zur Demenz verursachen

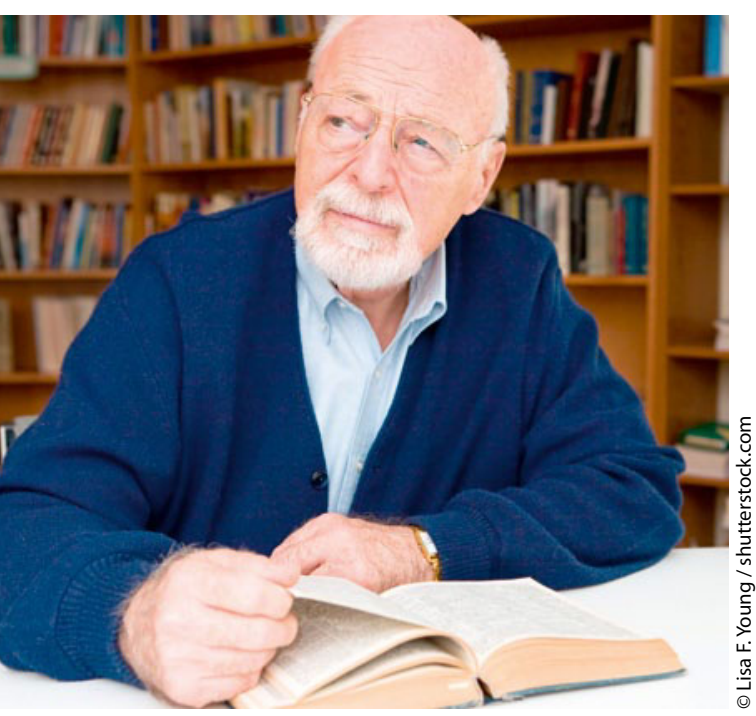

Effektive OAB-Therapie und trotzdem fit im Kopf können. Eine Ausnahme bildet Fesoterodin (Toviaz ${ }^{\circledast}$ ): Die Substanz ist weniger lipophil als andere Antimuskarinika und durchdringt die Blut-Hirn-Schranke kaum. Gleichzeitig wird Fesoterodin aktiv aus dem ZNS ins Blut rücktransportiert. Die Folge sind deutlich geringere ZNS-Effekte bei effektiver Verbesserung der Blasenfunktion. Das zeigt die SOFIA-Studie, deren Daten PD Dr. Mathias Oelke, Hannover, vorstellte [Wagg A et al. EAU 2011; abstract 880].

Die zwölfwöchige Studie mit 800 Patienten über 65 Jahre liefert erstmals Daten zur Wirksamkeit und Sicherheit in dieser Altersgruppe. Unter der flexiblen Dosis von $4 \mathrm{mg}$ oder $8 \mathrm{mg}$ reduzierten sich Drangereignisse gegenüber Baseline signifikant um 3,5 Episoden verglichen mit 1,9 Episoden unter Placebo. „Unabhängig vom Alter der Patienten und dem Zeitpunkt der Einnahme morgens oder abends - war die Wirkung immer gleich", so Oelke. Die Miktionsfrequenz verringerte sich signifikant und klinisch relevant um 1,9 versus 0,9 in der Kontrollgruppe. Bei Dranginkontinenzepisoden war die Wirkung numerisch, aber nicht signifikant besser. $70 \%$ der Patienten berich- teten, dass sich ihr Blasenzustand verbessert hat. Auch die Lebensqualität verbesserte sich signifikant und die Symptomlast verringerte sich. An die verblindete Phase schloss sich eine offene Phase von weiteren zwölf Monaten mit Fesoterodin für alle Patienten an. An deren Ende erreichten Patienten, die nach zwölf Wochen unter Placebo auf das Verum wechselten, identische Ergebnisse.

Häufige Nebenwirkungen waren Mundtrockenheit (34\%) und Obstipation (9\%). Therapieabbrüche wegen Harnverhalt waren nicht häufiger als unter Placebo. Hatten frühere Studien mit Anticholinergika deutliche kognitive Einbußen für behandelte Patienten gezeigt, waren die Veränderungen in der SOFIAStudie nur minimal. Michael Koczorek

Meet the Experts „Die Therapie der überak tiven Blase: (K)eine Frage des Alters?", im Rahmen des 63. DGU-Kongresses, Hamburg, 15. September 2011

Veranstalter: Pfizer, Berlin 